\title{
Fractals in Trade Duration: Capturing Long-Range Dependence and Heavy Tailedness in Modeling Trade Duration
}

\author{
Wei Sun \\ Institute for Statistics and Mathematical Economics \\ University of Karlsruhe, Germany \\ Svetlozar Rachev \\ Institute for Statistics and Mathematical Economics \\ University of Karlsruhe, Germany \\ and \\ Department of Statistics and Applied Probability \\ University of California, Santa Barbara, USA \\ Frank J. Fabozzi* \\ Yale School of Management, USA \\ Petko S. Kalev \\ Department of Accounting and Finance \\ Monash University, Australia
}

\footnotetext{
${ }^{*}$ Corresponding author: Frank J. Fabozzi, 858 Tower View Circle, New Hope, PA 18938, U.S.A. Email: FABOZZI321@aol.com. S. Rachev's research was supported by grants from Division of Mathematical, Life and Physical Science, College of Letters and Science, University of California, Santa Barbara, and the Deutschen Forschungsgemeinschaft. W. Sun's research was supported by grants from the Deutschen Forschungsgemeinschaft. P.S. Kalev's research was supported with a NCG grant from the Faculty of Business and Economics, Monash University. Data are supplied by Securities Industry Research Center of Asia-Pacific (SIRCA) on behalf of Reuters.
} 


\title{
Fractals in Trade Duration: Capturing Long-Range Dependence and Heavy Tailedness in Modeling Trade Duration
}

\begin{abstract}
Several studies that have investigated a few stocks have found that the spacing between consecutive financial transactions (referred to as trade duration) tend to exhibit long-range dependence, heavy tailedness, and clustering. In this study, we empirically investigate whether a larger sample of stocks exhibit those characteristics. Comparing goodness of fit in modeling trade duration data for stable distribution and fractional stable noise based on a procedure applying bootstrap methods developed by the authors, the empirical results in this paper suggest that the fractional stable noise and stable distribution dominate alternative distributional assumptions (i.e., lognormal distribution, fractional Gaussian noise, exponential distribution, and Weibull distribution) in our study.
\end{abstract}

Key Words: fractional stable noise, point processes, self-similarity, stable distribution, trade duration

JEL Classification: C41, G14 


\section{Introduction}

There is considerable interest in the information content and implications of the spacing between consecutive financial transactions (referred to as trade duration) for trading strategies and intra-day risk management. Market microstructure theory, supported by empirical evidence, suggests that the spacing between trades be treated as a variable to be explained or predicted since time carries information and closely correlates with price volatility (see, Bauwens and Veredas (2004), Diamond and Varrecchia (1987), Engle (2000), Engle and Russell (1998), Hasbrouck (1996), and O'Hara (1995)). Manganelli (2005) finds that returns and volatility directly interact with trade duration and trade order size. Trade durations tend to exhibit long-range dependence, heavy tailedness, and clustering (see, Bauwens and Giot (2000), Dufour and Engle (2000), Engle and Russell (1998), and Jasiak (1998)).

These findings raise two questions that we address in this paper:

1. Can single stochastic processes which capture long-range dependence and heavy tailedness be used in modeling trade duration data?

2. Can a relatively "powerful" distributional assumption in a relatively "simple" functional structure be used for efficient modeling of trade duration data?

It is necessary to treat long-range dependence, heavy tailedness, and clustering simultaneously in order to obtain more accurate predictions. Rachev and Mittnik (2000) note that for modeling financial data, not only does model structure play an important role, but distributional assumptions influence the modeling accuracy. The stable Paretian distribution ${ }^{1}$ can be used to capture characteristics of trade duration since it is rich enough to encompass those stylized facts in such data, such as non-Guassian, heavy tails, long-range dependence, and clustering. Other researchers have shown the advantages of stable distributions in financial modeling (see, Fama (1963), Mittnik and Rachev (1993a, 1993b), Rachev (2003), and Rachev et al. (2005)). Meanwhile several studies have reported that long-range dependence, self-similar processes, and stable distribution are very closely related (see, Samorodnitsky and Taqqu (1994), Rachev and Mittnik (2000), Rachev and Samorodnitsky (2001), Doukhan et al. (2003), Racheva and Samorodnitsky (2003), and Rachev et al. (2005)).

The Hurst index is also used to model long-range dependence, see Hurst (1951,1955). It quantifies the degree of long-range dependence and measures the self-similarity scaling. Fortunately, one type of self-similar process can possess the Hurst index and stable distribution together (i.e., can capture both long-range dependence and heavy tailedness). This kind of stochastic process is a fractional stable noise generated from fractional stable motion (see, Samorodnitsky and Taqqu (1994)). Therefore, single stochastic processes can capture long-range dependence and heavy tailedness, answering the first question posed above. Based on estimating intensity of point processes, an autoregressive conditional duration (ACD) model is proposed by Engle and Russell (1998) for modeling trade duration with intertemporal correlation. The ACD model is a joint approach combining transition analysis and Engle's

\footnotetext{
${ }^{1}$ To distinguish between Gaussian and non-Gaussian stable distribution, the latter is usually named stable Paretian distribution or Lévy stable distribution. Referring to it as a stable Paretian distribution highlights the fact that the tails of the non-Gaussian stable density have Pareto power-type decay and Lévy stable is the recognition of pioneering works done by Paul Lévy to the characterization of non-Gaussian stable laws (see Rachev and Mittnik (2000)).
} 
autoregressive conditional heteroscedasticity (ARCH) model. The motivation behind the ACD and the $\mathrm{ARCH}$ models is that in financial market events tend to occur in clusters. If fractional stable noise can be subordinated into the functional structure of the ACD model, then the second question can be answered.

In order to answer the two questions posed above, this paper introduces fractional stable noise as the single stochastic processes to model trade duration. In the empirical analysis of this paper, fractional stable noise is subordinated to the ACD model to model trade duration. As to self-similar processes, other single stochastic processes, such as fractional Gaussian noise which captures longrange dependence, are also is introduced as an alternative. Since the stable distribution itself can capture heavy tailedness and long-range dependence, we propose it as an alternative distribution that can better explain trade duration. In the empirical analysis, stable distribution is also subordinated to the ACD model. Some other distributions that are often used in modeling trade duration, such as lognormal distribution, exponential distribution and Weibull distribution, have been selected as alternative distributional assumptions in order to compare goodness of fit with the stable distribution and fractional stable noise. Utilizing two test statistics usually used to evaluate model performance under heavy-tailed assumptions, we examine trade duration for a sample of stocks to compare which distributional assumption fits better. By applying a newly developed test procedure that we formulate, based on a bootstrap method, we obtain empirical results that suggest the fractional stable noise and stable distribution dominate these alternative assumptions with high statistical significance. Comparing goodness of fit in the modeling of trade duration data for stable distribution and fractional stable noise, the empirical results indicate that the ACD model with stable distribution fits better than other combinations, while fractional stable noise itself fits better for the time series of trade duration.

The paper is organized as follows. In Section 2, we introduce two self-similar processes: fractional Gaussian noise and fractional stable noise. The method for estimating the parameters in the underlying process is introduced in Section 3. In Section 4, the methods of simulating fractional Gaussian noise and fractional stable noise are introduced. The empirical study based on trade duration data for 18 of the component stocks of the Dow Jones is reported in Section 5. In that section, we compare the goodness of fit of model under fractional stable noise and stable distribution together with distributions (the lognormal distribution, fractional Gaussian noise, exponential distribution, and Weibull distribution). We summarize our conclusions in Section 6.

\section{Specification of the self-similar processes}

Self-similarity is defined by Samorodnitsky and Taqqu (1994) as follows. Let $T$ be either $R, R_{+}=\{t$ : $t \geq 0\}$ or $\{t: t>0\}$. The real-valued process $\{X(t), t \in T\}$ is self-similar with Hurst index $H>0$ $(H$-ss $)$ if for any $a>0$ and $d \geq 1, t_{1}, t_{2}, \ldots, t_{d} \in T$, satisfying:

$$
\left(X\left(a t_{1}\right), X\left(a t_{2}\right), \ldots, X\left(a t_{d}\right)\right) \stackrel{d}{=}\left(a^{H} X\left(t_{1}\right), a^{H} X\left(t_{2}\right), \ldots a^{H} X\left(t_{d}\right)\right) .
$$

\subsection{Fractional Gaussian noise}

Mandelbrot and Wallis (1968) first introduced the fractional Brownian motion (FBM) and Samorodnitsky and Taqqu (1994) clarify the definition of FBM as a Gaussian process having self-similarity index 
$H$ and stationary increments. Mandelbrot and van Ness (1968) defined the stochastic representation

$$
B_{H}(t):=\frac{1}{\Gamma\left(H+\frac{1}{2}\right)}\left(\int_{-\infty}^{0}\left[(t-s)^{H-\frac{1}{2}}-(-s)^{H-\frac{1}{2}}\right] d B(s)+\int_{0}^{t}(t-s)^{H-\frac{1}{2}} d B(s)\right)
$$

where $\Gamma(\cdot)$ represents the Gamma function:

$$
\Gamma(a):=\int_{0}^{\infty} x^{a-1} e^{-x} d x
$$

and $0<H<1$ is the Hurst parameter. The integrator $B$ is the ordinary Brownian motion. The main difference between fractional Brownian motion and ordinary Brownian motion is that the increments in Brownian motion are independent while in fractional Brownian motion they are dependent. As to the fractional Brownian motion, Samorodnitsky and Taqqu (1994) define its increments $\left\{Y_{j}, j \in Z\right\}$ as fractional Gaussian noise (FGN), which is, for $j=0, \pm 1, \pm 2, \ldots, Y_{j}=B_{H}(j-1)-B_{H}(j)$.

\subsection{Fractional stable noise}

Fractional Brownian motion can capture the effect of long-range dependence, but with less power to capture the heavy tailedness. The existence of abrupt discontinuities in financial data, combined with the empirical observation of sample excess kurtosis and unstable variance, confirms the stable Paretian hypothesis in Mandelbrot $(1963,1983)$. It is natural to introduce stable Paretian distribution in selfsimilar processes in order to capture both long-range dependence and heavy tailedness. Samorodinitsky and Taqqu (1994) introduce the $\alpha$-stable $H$-sssi processes $\{X(t), t \in R\}$ with $0<\alpha<2$. If $0<\alpha<1$, the values of Hurst parameter are $H \in(0,1 / \alpha]$ and if $1<\alpha<2$, the values of Hurst parameter are $H \in(0,1]$. There are many different extensions of fractional Brownian motion to the stable distribution. The most commonly used is the linear fractional stable motion (also called linear fractional Lévy motion), $\left\{L_{\alpha, H}(a, b ; t), t \in(-\infty, \infty)\right\}$, which is defined by Samorodinitsky and Taqqu (1994) as follows:

$$
L_{\alpha, H}(a, b ; t):=\int_{-\infty}^{\infty} f_{\alpha, H}(a, b ; t, x) M(d x),
$$

where

$$
f_{\alpha, H}(a, b ; t, x):=a\left((t-x)_{+}^{H-\frac{1}{\alpha}}-(-x)_{+}^{H-\frac{1}{\alpha}}\right)+b\left((t-x)_{-}^{H-\frac{1}{\alpha}}-(-x)_{-}^{H-\frac{1}{\alpha}}\right),
$$

and where $a, b$ are real constants, $|a|+|b|>1,0<\alpha<2,0<H<1, H \neq 1 / \alpha$ and $M$ is an $\alpha$ stable random measure on $R$ with Lebesgue control measure and skewness intensity $\beta(x), x \in(-\infty, \infty)$ satisfying: $\beta(\cdot)=0$ if $\alpha=1$. Samorodinitsky and Taqqu (1994) define linear fractional stable noise expressed by $Y(t)$, and $Y(t)=X_{t}-X_{t-1}$,

$$
\begin{aligned}
Y(t) & =L_{\alpha, H}(a, b ; t)-L_{\alpha, H}(a, b ; t-1) \\
& =\int_{R}\left(a\left[(t-x)_{+}^{H-\frac{1}{\alpha}}-(t-1-x)_{+}^{H-\frac{1}{\alpha}}\right]\right. \\
& \left.+b\left[(t-x)_{-}^{H-\frac{1}{\alpha}}-(t-1-x)_{-}^{H-\frac{1}{\alpha}}\right]\right) M(d x)
\end{aligned}
$$

where $L_{\alpha, H}(a, b ; t)$ is linear fractional stable motion defined by equation (3), and $M$ is a stable random measure with Lebesgue control measure given $0<\alpha<2 .^{2}$ In this paper, if there is no special indication, fractional stable noise (fsn) is generated from linear fractional stable motion.

\footnotetext{
${ }^{2}$ Some properties of these processes have been discussed in Mandelbrot and Van Ness (1968), Maejima and Rachev (1987), Manfields et al. (2001), Rachev and Mittnik (2000), Rachev and Samorodnitsky (2001), Racheva and Samorodnitsky (2003), Samorodnitsky (1994, 1996, 1998), and Samorodinitsky and Taqqu (1994).
} 


\section{Estimation in self-similar processes}

\subsection{Estimating the self-similarity parameter in fractional Gaussian noise}

Beran (1994) discusses the Whittle estimator of self-similarity parameter. For fractional Gaussian noise, $Y_{t}$, let $f(\lambda ; H)$ denote the power spectrum of $Y$ after being normalized to have variance 1 and let $I(\lambda)$ the periodogram of $Y_{t}$; that is

$$
I(\lambda)=\frac{1}{2 \pi N}\left|\sum_{t=1}^{N} Y_{t} e^{i t \lambda}\right|^{2} .
$$

The Whittle estimator of $H$ is to find $\hat{H}$ that minimizes

$$
g(\hat{H})=\int_{-\pi}^{\pi} \frac{I(\lambda)}{f(\lambda ; \hat{H})} d \lambda .
$$

\subsection{Estimating the self-similarity parameter in fractional stable noise}

Stoev et al (2002) proposed the least-squares (LS) estimator of the Hurst index based on the finite impulse response transformation (FIRT) and wavelet transform coefficients of the fractional stable motion. A FIRT is a filter $v=\left(v_{0}, v 1, \ldots, v_{p}\right)$ of real numbers $v_{t} \in \Re, t=1, \ldots, p$, and length is $p+1$. It is defined for $X_{t}$ by

$$
T_{n, t}=\sum_{i=0}^{p} v_{i} X_{n(i+t)}
$$

where $n \geq 1$ and $t \in N$. The $T_{n, t}$ are the FIRT coefficients of $X_{t}$ (i.e., the FIRT coefficients of the fractional stable motion). The indices $n$ and $t$ can be explained as "scale" and "location". If $\sum_{i=0}^{p} i^{r} v_{i}=0$, for $r=0, \ldots, q-1$, but $\sum_{i=0}^{p} i^{q} v_{i} \neq 0$, the filter $v_{i}$ can be said to have $q \geq 1$ zero moments. If $\left\{T_{n, t}, n \geq 1, t \in N\right\}$ is the FIRT coefficients of fractional stable motion with the filter $v_{i}$ that has at least one zero moment, Stoev et al (2002) prove the following properties of $T_{n, t}$ : (1), $T_{n, t+h} \stackrel{d}{=} T_{n, t}$, and (2), $T_{n, t} \stackrel{d}{=} n^{H} T_{1, t}$, where $h, t \in N, n \geq 1$. We assume that $T_{n, t}$ are available for the fixed scales $n_{j} j=1, \ldots, m$ and locations $t=0, \ldots, M_{j}-1$ at the scale $n_{j}$, since only a finite number, say $M_{j}$, of the FIRT coefficients are available at the scale $n_{j}$. By using these properties, we have

$$
E \log \left|T_{n_{j}, 0}\right|=H \log n_{j}+E \log \left|T_{1,0}\right| \text {. }
$$

The left-hand side of this equation can be approximated by

$$
Y_{\log }\left(M_{j}\right)=\frac{1}{M_{j}} \sum_{t=0}^{M_{j}-1} \log \left|T_{n_{j}, t}\right| .
$$

Then we get

$$
\left(\begin{array}{c}
Y_{\log }\left(M_{1}\right) \\
\vdots \\
Y_{\log }\left(M_{m}\right)
\end{array}\right)=\left(\begin{array}{cc}
\log n_{1} & 1 \\
\vdots & \vdots \\
\log n_{m} & 1
\end{array}\right)\left(\begin{array}{c}
H \\
E \log \left|T_{1,0}\right|
\end{array}\right)+\left(\begin{array}{c}
\sqrt{M}\left(Y_{\log }\left(M_{1}\right)-E \log \left|T_{n_{1}, 0}\right|\right) \\
\vdots \\
\sqrt{M}\left(Y_{\log }\left(M_{m}\right)-E \log \left|T_{n_{m}, 0}\right|\right)
\end{array}\right) .
$$

We can express above equation as follows

$$
Y=X \theta+\frac{1}{\sqrt{M}} \varepsilon
$$


where $\varepsilon$ is the vector showing the difference between $\sqrt{M} Y_{\log }\left(M_{m}\right)$ and $\sqrt{M} E\left(\log \left|T_{n_{m}, 0}\right|\right)$. Equation (10) shows that the self-similarity parameter $H$ can be estimated by a standard linear regression of the vector $Y$ against the matrix $X$. Stoev et al. (2002) show the details for implementing such a procedure.

\subsection{Estimating the parameters of the stable Paretian distribution}

The stable distribution requires four parameters for complete description: an index of stability $\alpha \in$ $(0,2]$ also called the tail index, a skewness parameter $\beta \in[-1,1]$, a scale parameter $\gamma>0$, and a location parameter $\zeta \in \Re$. There is unfortunately no closed-form expression for the density function and distribution function of a stable distribution. Rachev and Mittnik (2000) give the definition for the stable distribution: A random variable $X$ is said to have a stable distribution if there are parameters $0<\alpha \leq 2,-1 \leq \alpha \leq 1, \gamma \geq 0$ and $\zeta$ real such that its characteristic function has the following form:

$$
E \exp (i \theta X)=\left\{\begin{array}{rlr}
\exp \left\{-\gamma^{\alpha}|\theta|^{\alpha}\left(1-i \beta(\sin \theta) \tan \frac{\pi \alpha}{2}\right)+i \zeta \theta\right\}, & \text { if } \quad \alpha \neq 1 \\
\exp \left\{-\gamma|\theta|\left(1+i \beta \frac{2}{\pi}(\sin \theta) \ln |\theta|\right)+i \zeta \theta\right\}, & \text { if } \quad \alpha=1
\end{array}\right.
$$

and,

$$
\sin \theta=\left\{\begin{aligned}
1, & \text { if } \quad \theta>0 \\
0, & \text { if } \quad \theta=0 \\
-1, & \text { if } \quad \theta<0
\end{aligned}\right.
$$

Stable density is not only support for all of $(-\infty,+\infty)$, but also for a half line. For $0<\alpha<1$ and $\beta=1$ or $\beta=-1$, the stable density is only for a half line.

In order to estimate the parameters of the stable distribution, the maximum likelihood estimation (MLE) method given in Rachev and Mittnik (2000) has been employed. Given $N$ observations, $X=$ $\left(X_{1}, X_{2}, \cdots, X_{N}\right)^{\prime}$ for the positive half line the log-likelihood function is of the form

$$
\ln (\alpha, \lambda ; X)=N \ln \lambda+N \ln \alpha+(\alpha-1) \sum_{i=1}^{N} \ln X_{i}-\lambda \sum_{i=1}^{N} X_{i}^{\alpha},
$$

which can be maximized using, for example, a Newton-Raphson algorithm. It follows from the first-order condition,

$$
\lambda=N\left(\sum_{i=1}^{N} X_{i}^{\alpha}\right)^{-1}
$$

that the optimization problem can be reduced to finding the value for $\alpha$ which maximizes the concentrated likelihood

$$
\ln ^{*}(\alpha ; X)=\ln \alpha+\alpha \nu-\ln \left(\sum_{i=1}^{N} X_{i}^{\alpha}\right)
$$

where $\nu=N^{-1} \Sigma_{i=1}^{N} \ln X_{i}$. The information matrix evaluated at the maximum likelihood estimates, denoted by $I(\hat{\alpha}, \hat{\lambda})$, is given by

$$
I(\hat{\alpha}, \hat{\lambda})=\left(\begin{array}{cc}
N \hat{\alpha}^{-2} & \sum_{i=1}^{N} X_{i}^{\hat{\alpha}} \ln X_{i} \\
\sum_{i=1}^{N} X_{i}^{\hat{\alpha}} \ln X_{i} & N \hat{\lambda}^{-2}
\end{array}\right)
$$


It can be shown that, under fairly mild condition, the maximum likelihood estimates $\hat{\alpha}$ and $\hat{\lambda}$ are consistent and have asymptotically a multivariate normal distribution with mean $(\alpha, \lambda)^{\prime}$ (see Rachev and Mittnik (2000)).

Other methods for estimating the parameters of a stable distribution (i.e., the method of moments based on the characteristic function, the regression-type method, and the fast Fourier transform method) are discussed in Stoyanov and Racheva-Iotova (2004a, 2004b, 2004c).

\section{Simulation of self-similar processes}

\subsection{Simulation of fractional Gaussian noise}

Paxson (1997) gives a method to generate the fractional Gaussian noise by using the Discrete Fourier Transform of the spectral density. Bardet et al. (2003) give a concrete simulation procedure based on this method with respect to alleviating some of the problems faced in practice. The procedure is:

1. Choose an even integer $M$. Define the vector of the Fourier frequencies $\Omega=\left(\theta_{1}, \ldots, \theta_{M / 2}\right)$, where $\theta_{t}=2 \pi t / M$ and compute the vector $F=f_{H}\left(\theta_{1}\right), \ldots, f_{H}\left(\theta_{M / 2}\right)$, where

$$
f_{H}(\theta)=\frac{1}{\pi} \sin (\pi H) \Gamma(2 H+1)(1-\cos \theta) \sum_{t \in \aleph}|2 \pi t+\theta|^{-2 H-1}
$$

$f_{H}(\theta)$ is the spectral density of FGN.

2. Generate $M / 2$ i.i.d exponential $\operatorname{Exp}(1)$ random variables $E_{1}, \ldots, E_{M / 2}$ and $M / 2$ i.i.d uniform $U[0,1]$ random variables $U_{1}, \ldots, U_{M / 2}$.

3. Compute $Z_{t}=\exp \left(2 i \pi U_{t}\right) \sqrt{F_{t} E_{t}}$, for $t=1, \ldots, M / 2$.

4. Form the $M$-vector: $\tilde{Z}=\left(0, Z_{1}, \ldots Z_{(M / 2)-1}, Z_{M / 2}, \bar{Z}_{(M / 2)-1}, \ldots, \bar{Z}_{1}\right)$.

5. Compute the inverse FFT of the complex $Z$ to obtain the simulated sample path.

\subsection{Simulation of fractional stable noise}

Replacing the integral in equation (5) with a Riemann sum, Stoev and Taqqu (2004) generate the approximation of fractional stable noise. They introduce parameters $n, N \in \aleph$, and let the fractional stable noise $Y(t)$ expressed as

$$
Y_{n, N}(t):=\sum_{j=1}^{n N}\left(\left(\frac{j}{n}\right)_{+}^{H-1 / \alpha}-\left(\frac{j}{n}-1\right)_{+}^{H-1 / \alpha}\right) L_{\alpha, n}(n t-j),
$$

where $L_{\alpha, n}(t):=M_{\alpha}((j+1) / n)-M_{\alpha}(j / n), j \in \Re$. The parameter $n$ is mesh size and the parameter $M$ is the cut-off of the kernel function. Stoev and Taqqu (2004) describe an efficient approximation involving the Fast Fourier Transformation algorithm for $Y_{n, N}(t)$. Consider the moving average process $Z(m), m \in \aleph$,

$$
Z(m):=\sum_{j=1}^{n M} g_{H, n}(j) L_{\alpha}(m-j)
$$


where

$$
g_{H, n}(j):=\left(\left(\frac{j}{n}\right)^{H-1 / \alpha}-\left(\frac{j}{n}-1\right)_{+}^{H-1 / \alpha}\right) n^{-1 / \alpha}
$$

and where $L_{\alpha}(j)$ is the series of i.i.d standard stable Paretian random variables. Since $L_{\alpha, n}(j) \stackrel{d}{=}$ $n^{-1 / \alpha} L_{\alpha}(j), j \in \Re$, equations (18) and (19) imply $Y_{n, N}(t) \stackrel{d}{=} Z(n t)$, for $t=1, \ldots, T$. Then, the computing of $Y_{n, N}(t)$ is moved to focus on the moving average series $Z(m), m=1, \ldots, n T$. Let $\tilde{L}_{\alpha}(j)$ be the $n(N+T)$-periodic with $\tilde{L}_{\alpha}(j):=L_{\alpha}(j)$, for $j=1, \ldots, n(N+T)$ and let $\tilde{g}_{H, n}(j):=g_{H, n}(j)$, for $j=$ $1, \ldots, n N ; \tilde{g}_{H, n}(j):=0$, for $j=n N+1, \ldots, n(N+T)$. Then

$$
\{Z(m)\}_{m=1}^{n T} \stackrel{d}{=}\left\{\sum_{j=1}^{n(N+T)} \tilde{g}_{H, n}(j) \tilde{L}_{\alpha}(n-j)\right\}_{m=1}^{n T},
$$

because for all $m=1, \ldots, n T$, the summation in equation (19) involves only $L_{\alpha}(j)$ with indices $j$ in the range $-n N \leq j \leq n T-1$. Using a circular convolution of the two $n(N+T)$-periodic series $\tilde{g}_{H, n}$ and $\tilde{L}_{\alpha}$ computed by using their Discrete Fourier Transforms (DFT), the variables $Z(n), m=1, \ldots, n T$ (i.e., the fractional stable noise), can be generated.

\section{Empirical study}

In this section, we report the results of empirical tests that investigate the goodness of fit of several candidate distributional assumptions.

\subsection{The data}

Ultra-high frequency data of 18 Dow Jones index component stocks based on NYSE trading for year 2003 are examined. ${ }^{3}$ The companies in the sample are listed in Table 1. Our sample is considerably larger than other studies that have investigated trade duration. Table 2 lists those studies and the stocks included in each one. Note that for the studies that include U.S. stocks, IBM is included in 7 of 11 studies and because the sample size is small, IBM constitutes a major part of those studies. IBM is included in our study also.

The trade durations were calculated for regular trading hours (i.e., overnight trading was not considered). Consistent with Engle and Russell (1998) and Ghysels et al. (2004), open trades are deleted in order to avoid effects induced by the opening auction. Therefore trade durations only from 10:00 to 16:00 are considered.

Figures 1 to 6 plot several sampled trade duration series. These figures show data characteristics that are consistent with the data patterns reported in the literature. For the trade durations of each stock in our study, sample period runs were performed from 4 January 2003 to 31 December 2003. We will let $N$ denote the length of the sample, sub-sample series that have been randomly selected by a moving window with length $T(1 \leq T \leq N)$. Replacement is allowed in the sampling. Stoev and Taqqu (2004) suggest that $2^{14}-6,000=10,384$ is the optimal length for a fractional stable noise series to be

\footnotetext{
${ }^{3}$ The data from were provided by The Securities Industry Research Center of Asia-Pacific in Australia. The Dow Jones index consists of 30 stocks. The whole database we developed included data from 1996 to 2003 for stocks that remained in the index over the entire period. Only 18 stocks satisfied that requirement and we use the data of 2003 in this study.
} 
simulated efficiently. Therefore, in the empirical analysis, sub-sample length (i.e., the window length) of $T=10,384$ was chosen. A total of 684 sub-samples were randomly created.

The trade duration data are distributed asymetrically. All observations of duration are positive numbers. The stable distribution, fractional Gaussian noise, and fractional stable are all defined on both positive and negative supports. In our empirical study, we transfer the asymmetrically distributed series to the symmetrically distributed series before we estimate the corresponding parameters. Note that only the positive numbers of the generated series are considered in our simulation.

\subsection{Preliminary tests}

Table 1 shows the descriptive statistics of the trade duration data in our study. From the statistics reported in this table, it can be seen that excess kurtosis exists.

Engle (1982) proposes a Lagrange-multiplier test for ARCH phenomenon. A test statistic for ARCH of lag order $q$ is given by

$$
X_{q} \equiv n R_{q}^{2}
$$

where $R_{q}^{2}$ is the non-centered goodness-of-fit coefficient of a $q$ th order autoregression of the squared residuals taken from the original regression

$$
\hat{u}_{t}^{2}=\omega_{0}+\omega_{1} \hat{u}_{t-1}^{2}+\omega_{2} \hat{u}_{t-2}^{2}+\cdots+\omega_{q} \hat{u}_{t-q}^{2}+e_{t},
$$

where $\hat{u}$ is the residual in original regression equation. Under the null hypothesis of the residuals of the original model being normally i.i.d., the ARCH statistic of lag order $q$ follows a $\chi^{2}$ distribution with $q$ degree of freedom:

$$
\lim _{n \rightarrow \infty} X_{q} \sim \chi_{q}^{2}
$$

Table 3 shows the test statistics and critical values in which we can reject the null hypothesis that there is no ARCH effect at different lag levels for the duration increments. It is clear that an ARCH effect is exhibited in these data.

The Hurst index $H \in(0,1)$ usually serves as the measure of the tendency of a process and stands for the self-similarity index in Gaussian stochastic processes. It can be somewhat explained by considering the covariance of two consecutive increments. When $H \in(0,0.5)$, the increments of a process tend to have opposite signs and thus are more zigzagging due to the negative covariance; when $H \in(0.5,1)$, the covariance between these two increments is positive and less zigzagging of the process; when $H=0.5$, the covariance between this two increments is zero. It can be stated as follows: If the Hurst index is less than 0.5 , the process displays "anti-persistence" which means that the positive excess return is more likely to be reversed and the performance in the next period is likely to be below the average, or on the contrary, the negative excess return is more likely to be reversed and the performance in the next period is likely to be above the average. If the Hurst index is greater than 0.5 , the process displays "persistence" which means that the positive excess return or the negative excess return is more likely to be continued and the performance in the next period is likely to be the same as that in the current period. If the Hurst index is equal to 0.5 , the process displays no memory, which means the performance 
in the next period has equal probability to be below and above the performance in the current period. From Table 1, we find that the Hurst index has no value of 0.5 , which indicates that the memory effect occurs in our samples.

The Hurst index for non-Gaussian stable processes has different bounds for "persistence" and "antipersistence". For tail index $\alpha \in(0,2)$, when $H \in(0,1 / \alpha)$, the processes exhibit "anti-persistence", and when $H \in(1 / \alpha, 1)$, the processes exhibit "persistence". There is no long-range dependence when $\alpha \in(0,1]$ because the Hurst index is bounded in the interval $(0,1)$. When $H=1 / \alpha$, depending on the value of $\alpha$ the processes exhibit either no memory or long-range dependence. ${ }^{4}$ From Table 1 , we find that the Hurst index has no value of $1 / \alpha$. Therefore, we find that long-range dependence occurs in our samples.

We use the Ljung-Box-Pierce $Q$-statistic based on autocorrelation function to test the serial correlation (i.e., the memory effect). The $Q$-statistic is given as follows:

$$
Q: \sim \chi_{m}^{2}=N(N+2) \sum_{k=1}^{m} \frac{\rho_{k}^{2}}{N-k},
$$

where, $N$ denotes the sample size, $m$ the number of autocorrelation lags included in the statistic, and $\rho_{k}$ the sample autocorrelation at lag order $k$ which is

$$
\rho_{k}=\frac{\sum_{t=1}^{N-k} y_{t} y_{t+k}}{\sum_{t=1}^{N} y_{t}^{2}} .
$$

Ljung and Box (1978) show that the $Q$-statistic follows the asymptotic chi-square distribution with $m$ degrees of freedom.

Table 4 shows that the null hypothesis that there is no serial correlation can be rejected at different lags. This table shows that the memory effect occurs in each duration series. In order to see when the memory effect vanishes, we compare the $Q$-statistic with its corresponding critical value. When the quotation of the $Q$-statistic and the corresponding critical value are less than 1 , we cannot reject the null hypothesis that there is no serial correlation. Table 4 also shows such quotations. From this table, all the trade durations exhibit serial correlation. After 500 lags, the memory effect vanishes for 7 stocks and after 1500 lags, the memory effect vanishes for 13 stocks. From the ratios in Table 4, we find that the speed of autocorrelation decay is declining, which confirms the effect of long-range dependence.

\subsection{The methodology of finding the best model}

In our empirical study, we simulate a series for each distributional assumption with and without subordinating them into the $\operatorname{ACD}(1,1)$ structure. Then we compare the goodness of fit of the simulated together with originial trade duration series.

The class of ACD model can be defined as:

$$
d_{i}=\sigma_{i} u_{i}
$$

and

$$
\sigma_{t}^{2}=\kappa+\sum_{i=1}^{p} \gamma_{i} d_{t-i}+\sum_{j=1}^{q} \theta_{j} \sigma_{t-j}^{2},
$$

\footnotetext{
${ }^{4}$ A detailed discussion see Samorodnitsky and Taqqu (1994) and Racheva-Iotova and Samorodnitsky (2003).
} 
$u_{t}$ can be calculated from $d_{t} / \sigma_{t}$. We define

$$
\tilde{u}_{t}=\frac{d_{t}}{\hat{\sigma}_{t}}
$$

where $\hat{\sigma}_{t}$ is the estimation of $\sigma_{t}$. In our empirical analysis, an $\operatorname{ACD}(1,1)$ model structure is adopted. The objective is to check the statistical characteristics exhibited by trade duration $d_{t}$ and the error term $\tilde{u_{t}}$ in $\operatorname{ACD}(1,1)$ structure. We simulate $d_{t}$ and $\tilde{u}_{t}$ with the $\operatorname{ACD}(1,1)$ structure based on the parameters estimated from the empirical series. Then we test the goodness of fit between the empirical series and the simulated series. Six candidate distributional assumptions - lognormal distribution, stable distribution, exponential distribution, Weibull distribution, fractional Gaussian noise, and fractional stable noise - are analyzed for estimation, simulation, and testing.

The Kolmogorov-Simirnov distance (KS) and Anderson-Darling distance (AD) proposed by Rachev and Mittnik (2000) are used as the criterion for the goodness of fit testing. They are defined as following:

$$
K S=\sup _{x \in \Re}\left|F_{s}(x)-\tilde{F}(x)\right|,
$$

and

$$
A D=\sup _{x \in \Re} \frac{\left|F_{s}(x)-\tilde{F}(x)\right|}{\sqrt{\tilde{F}(x)(1-\tilde{F}(x))}},
$$

where $F_{s}(x)$ denotes the empirical sample distribution and $\tilde{F}(x)$ is the estimated distribution function. The major disadvantage of KS statistic researchers have argued is that it tends to be more sensitive near the center of the distribution than at the tails. But AD statistic can overcome this. The reliability of testing the empirical distribution will be increased with the help of these two statistics, with the KS distance focusing on the deviations around the median of the distribution and the AD distance on the discrepancies in the tails.

\subsection{Results}

The $\mathrm{AD}$ and KS statistics are calculated for the six candidate distributional assumptions. Table 5 reports the descriptive statistics of the computed AD and KS statistics. From Table 5, fractional stable noise and stable distribution exhibit a smaller mean value for the $\mathrm{AD}$ and KS statistics in comparison with the other four distributions. Figure 2 shows the boxplot of AD statistics of $\tilde{u}_{t}$ for the six alternative distributional assumptions investigated. Figure 3 shows the boxplot of AD statistics for $d_{t}$. Figure 4 shows the boxplot of KS statistics of $\tilde{u_{t}}$ for the six alternative distributional assumptions. Figure 5 shows the boxplot of KS statistics of $d_{t}$. These figures show that fractional stable noise and stable distribution have a small value of $\mathrm{AD}$ and KS statistics, confirming the results reported in Table 5. These results indicate that with or without an $\operatorname{ACD}(1,1)$ model structure, the fractional stable noise and stable distribution perform better than the other four tested distributional assumptions based on the criterion for goodness of fit testing.

From Figures 2 to 5 , we can see that the fractional stable noise and the stable distribution fit $\tilde{u}_{t}$ and $d_{t}$ better than other distributional assumptions. In order to empirically examine our conjecture, we formulate a statistical test procedure. Because we know that smaller AD and KS statistics mean 
better goodness of fit, in our test we are going to statistically test how significantly "smaller" AD and KS statistics are. The hypothesis test is:

$$
\begin{aligned}
& H_{0}: \mu_{\text {criterion } 1}-\mu_{\text {criterion } 2} \geq 0 \\
& H_{1}: \mu_{\text {criterion } 1}-\mu_{\text {criterion } 2}<0
\end{aligned}
$$

where $\mu_{\text {criterion }}$ is the mean value of $\mathrm{AD}$ or $\mathrm{KS}$ statistics of the candidate distributional assumptions investigated. The distributions of $\mathrm{AD}$ and $\mathrm{KS}$ values are unknown. All $\mathrm{AD}$ or KS values are expressed as i.i.d. random variables $X_{1}, X_{2}, \ldots, X_{n}$, each with distribution function $F_{X}(\cdot \mid \theta)$. A $100(1-\alpha) \%$ upper confidence bound (UCB) is defined as $U\left(X_{1}, X_{2}, \ldots, X_{n}\right)$ for a function of $h(\theta)$ if for every $\theta$,

$$
P_{\theta}\left(h(\theta) \leq U\left(X_{1}, X_{2}, \ldots, X_{n}\right)\right) \geq 1-\alpha
$$

and $\left(-\infty, U\left(X_{1}, X_{2}, \ldots, X_{n}\right)\right]$ is the $100(1-\alpha) \%$ upper confidence interval for $h(\theta)$. Similarly, $L\left(X_{1}\right.$, $\left.X_{2}, \ldots, X_{n}\right)$ is a $100(1-\alpha) \%$ lower confidence bound (LCB) for the function $h(\theta)$ for every $\theta$

$$
P_{\theta}\left(h(\theta) \geq L\left(X_{1}, X_{2}, \ldots, X_{n}\right)\right) \geq 1-\alpha
$$

and $\left[L\left(X_{1}, X_{2}, \ldots, X_{n}\right),+\infty\right)$ is the $100(1-\alpha) \%$ lower confidence interval for $h(\theta)$.

As hypothesis testing and confidence intervals are dual concepts, the hypothesis testing in (50) is in fact evaluated by following test rules; that is, (1) if UCB is less than zero, $H_{0}$ can be rejected, (2) if LCB is greater than zero, $H_{0}$ cannot be rejected, and (3) if $H_{0}$ is greater than LCB but at the same time less than UCB, there is no statistically significant conclusion. Employing the bootstrap method introduced in DiCiccio and Efron (1996), the 99\% bootstrap confidence intervals are reported in Table 6. From this table, at a high confidence level, fractional stable noise and stable distribution are more suitable to modeling trade duration data with or without support of an $\operatorname{ACD}(1,1)$ structure.

In comparing the fractional stable noise and stable distribution, it is unclear as to whether the fractional stable noise is better than the stable distribution or vice versa. Table 7 compares the supporting cases for the fractional stable noise and stable distribution. The fractional stable noise has a slightly better support than stable distribution. The stable distribution has a greater number of supporting cases in comparison to the fractional stable noise in modeling duration data with an $\mathrm{ACD}(1,1)$ structure.

\section{Conclusions}

The empirical research with very few stocks have demonstrated that trade duration data exhibit three characteristics: long-range dependence, heavy tailedness, and clustering. In this paper, we investigate the presence of these characteristics using a larger number of stocks and investigate whether for modeling trade duration data: (1) a single stochastic processes capturing long-range dependence and heavy tailedness and; (2) a relatively powerful distributional assumption in a relatively simple functional structure can be used.

To examine these issues, we introduce fractional stable noise and fractional Gaussian noise to capture long-range dependence and heavy tailedness in modeling the trade duration. In our empirical analysis, we investigate six distributional assumptions (fractional stable noise, fractional Gaussian noise, stable 
distribution, lognormal distribution, exponential distribution, and Weibull distribution) for modeling the trade duration for 18 Dow Jones index component stocks. By using parameters estimated from the empirical series, we simulate a series for each distributional assumption with and without subordinating them into an $\operatorname{ACD}(1,1)$ structure. Then we compare the goodness of fit for these generated series to the empirical series by adopting two test criteria for testing heavy-tailed distributions, the KolmogorovSimirnov and Anderson-Darling statistics. A test procedure is formulated based on a bootstrap method, and it is used in order to obtain empirical results.

The above test procedure yields empirical evidence which shows that the stable distribution and fractional stable noise are better in modeling trade duration than the exponential distribution, lognormal distribution, Weibull distribution, and fractional Gaussian noise. The results indicate that residuals from the $\operatorname{ACD}(1,1)$ model are more likely to be described by a stable distribution and trade durations exhibit the features of fractional stable noise. That is, stable distribution subordinated with an $\operatorname{ACD}(1,1)$ structure and fractional stable noise, demonstrate superior performance in the modeling of trade duration.

We argue that it is critical that the findings reported in this paper be taken into account in modeling trade duration. Many studies have found that stable distribution is a better description of financial data because it can capture heavy tailedness and has a close relationship with long-range dependence. As a self-similar process, fractional stable noise can capture almost all reported stylized facts in financial return data, such as heavy tailedness, long memory, non-Gaussian characters, and clustering. Therefore, if fractional stable noise and stable distribution can be properly employed in financial modeling, more accurate prediction might be realized by well-defined functional models.

\section{References}

[1] Bardet, J., G. Lang, G. Oppenheim, A. Philippe, and M. Taqqu: Generators of long-range dependent processes: a survey, In Theory and Applications of Long-range Dependence, P. Doulkhan, G. Oppenheim, and M. Taqqu (eds.), Birkhäuser: Boston (2003).

[2] Bauwens, L.: Econometric analysis of intra-daily trading activity on Tokyo stock exchange, IMES Discuussion Paper Series, 2005-E-3 (2005)

[3] — , and D. Veredas: , The stochastic conditional duration model: a latent variable model for the analysis of financial durations, Journal of Econometrics, 119, 381-412 (2004)

[4] - and P. Giot: The logarithmic ACD model: an application to the bid-ask quote process of three NYSE stocks, Annals d'Economie et de Statistique, 60,117-149 (2000)

[5] — : Asymmetric ACD models: introducing price information in ACD models, Empirical Economics, 28, 709-731 (2000)

[6] — J. Gramming, and D. Veredas: A comparison of financial duration models via density forecasts, International Journal of Forecasting, 20, 589-609 (2004)

[7] Beran, J: Statistics for Long-Memory Processes, Chapman \& Hall: New York (1994)

[8] Diamond, W. and R. Verrecchia: Constraints on short-selling and asset price adjustment to private information, Journal of Financial Economics, 18, 277-311 (1987)

[9] DiCiccio, T. J. and B. Efron: Bootstrap confidence intervals, Statistical Science, 11, 189-228 (1996) 
[10] Doukhan, P., G. Oppenheim and M. Taqqu: (eds.), Theory and Applications of Long-Range Dependence, Birkhäuser: Boston (2003)

[11] Dufour, A., and R. Engle: Time and the price impact of a trade, Journal of Finance,55, 2467-2498 (2000)

[12] Engle, R. F.: Autoregressive conditional heteroskedasticity with estimates of the variance of U.K. inflation, Econometrica, 50, 987-1008 (1982)

[13] — : The econometrics of ultra-high frequency data, Econometrica, 68, 1-22 (2000)

[14] — and J.R. Russell: Autoregressive conditional duration: a new model for irregularly spaced transaction data, Econometrica, 66, 1127-1162 (1998)

[15] Engle, R. and A. Lunde: Trades and quotes: a bivariate point process, Journal of Financial Econometrics, 1, 159-188 (2003)

[16] Fama, E.: Mandelbrot and the stable Paretian hypothesis, Journal of Business, 36, 420-429 (1963)

[17] Feng, D., G. J. Jiang and P. Song: Stochastic conditional duration models with "leverage effect" for financial transaction data, Journal of Financial Econometrics, 119, 413-433 (2004)

[18] Fernandes, M., and J. Gramming: A family of autoregressive conditional duration models, Ensaios Econmicos, 501 (2003)

[19] — N Nonparametric specification tests for conditional duration models, Journal of Econometrics, 127, 35-68 (2005)

[20] Ghysels, E., C. Gouriéroux and J. Jasiak: Stochastic volatility duration models, Journal of Econometrics, 119, 413-433 (2004)

[21] Hasbrouck, J.: Modeling microstructure time series, in Maddala G., and C. R Rao (eds.) Statistical Methods in Finance (Handbook of Statistics, Volume 14), North-Holland (1996)

[22] Hurst, H.: Long-term storage capacity of reservoirs, Transactions of the American Society of Civil Engineers, 116, $770-808(1951)$

[23] — : Methods of using long-term storage in reservoirs, Proceedings of the Institution of Civil Engineers, Part I, 519-577 (1955)

[24] Jasiak, J.: Persistence in intertrade durations, Finance, 19, 166-195 (1998)

[25] Mandelbrot, B. B.: New methods in statistical economics, Journal of Political Economy, 71, 421-440 (1963)

[26] — : The Fractal Geometry of Nature, Freeman: San Francisco (1983)

[27] — , and J. Van Ness: Fractional brownian motions, fractional noises and applications, SIAM Review, 10, 422-437 (1968)

[28] — , and J. Wallis: Noah, Joseph and operational hydrology, Water Resources Research, 4, 909-918 (1968)

[29] Maejima, M., and S. Rachev: An ideal metric and the rate of convergence to a self-similar process, Annals of Probability,15, 702-727 (1987)

[30] Manganelli, S.: Duration, volume and volatility impact of trades, Journal of Financial Markets, 8, 377-399 (2005)

[31] Mansfield, P., S. Rachev and G. Samorodnitsky: Long strange segments of a stochastic process and long-range dependence, The Annals of Applied Probability, 11, 878-921 (2001)

[32] Mittnik, S., and S. T. Rachev: Modeling asset returns with alternative stable models, Econometric Reviews, 12, 261-330 (1993a) 
[33] — : Reply to comments on "Modeling asset returns with alternative stable models" and some extensions, Econometric Reviews, 12, 347-389 (1993b)

[34] O'Hara, M.: Market Microstructure Theory, Blackwell: Cambridge (1995)

[35] Paxson, V.: Fast, approximate synthesis of fractional Gaussian noise for generating self-similar network traffic. Computer Communication Review, 27, 5-18 (1997)

[36] Rachev, S.: (edt.), Handbook of Heavy Tailed Distributions in Finance, Elsevier: Amsterdam (2003)

[37] — and S. Mittnik, (2000), Stable Paretian Models in Finance, Wiley: New York

[38] Rachev, S. and G. Samorodnitsky: Long strange segments in a long range dependent moving average, Stochastic Processes and their Applications, 93, 119-148 (2001)

[39] Rachev, S., C. Menn and F. Fabozzi: Fat-Tailed and Skewed Asset Return Distributions, Wiley: Hoboken (2005)

[40] Racheva, B. and G. Samorodnitsky: Long range dependence in heavy tailed stochastic processes, in Handbook of Heavy Tailed Distributions in Finance, S. Rachev, (edt.), Elsevier: Amsterdam (2003)

[41] Samorodnitsky, G.: Possible sample paths of self-similar alpha-stable processes, Statistics and Probability Letters, 19, 233-237 (1994)

[42] — : A class of shot noise models for financial applications, in Proceeding of Athens International Conference on Applied Probability and Time Series. Volume 1: Applied Probability, C. Heyde, Yu. V. Prohorov, R. Pyke, and S. Rachev, (eds.), Springer: Berlin (1996)

[43] — : Lower tails of self-similar stable processes, Bernoulli, 4, 127-142 (1998)

[44] — and M. S. Taqqu: Stable Non-Gaussian Random Processes: Stochastic Models with Infinite Variance, Chapman \& Hall/CRC: Boca Raton (1994)

[45] Stoev, S., V. Pipiras and M. Taqqu: Estimation of the self-similarity parameter in liner fractional stable motion, Signal Processing, 82, 1873-1901 (2002)

[46] Stoev, S., and M. Taqqu: Simulation methods for linear fractional stable motion and FARIMA using the Fast Fourier Transform, Fractals, 12, 95-121 (2004)

[47] Stoyanov, S., and B. Racheva-Iotova: Univariate stable laws in the field of finance-approximation of density and distribution functions, Journal of Concrete and Applicable Mathematics, 2, 38-57 (2004a)

[48] — : Univariate stable laws in the field of finance-parameter estimation, Journal of Concrete and Applicable Mathematics, 2, 24-49 (2004b)

[49] — : Numerical methods for stable modeling in financial risk management, in Handbook of Computational and Numerical Methods, edited by S. Rachev, Birkhauser (2004c)

[50] Zhang, M., J. Russell and R. Tsay: A nonlinear autoregressive conditional duration model with applications to financial transaction data, Journal of Econometrics, 104, 179-207 (2001) 


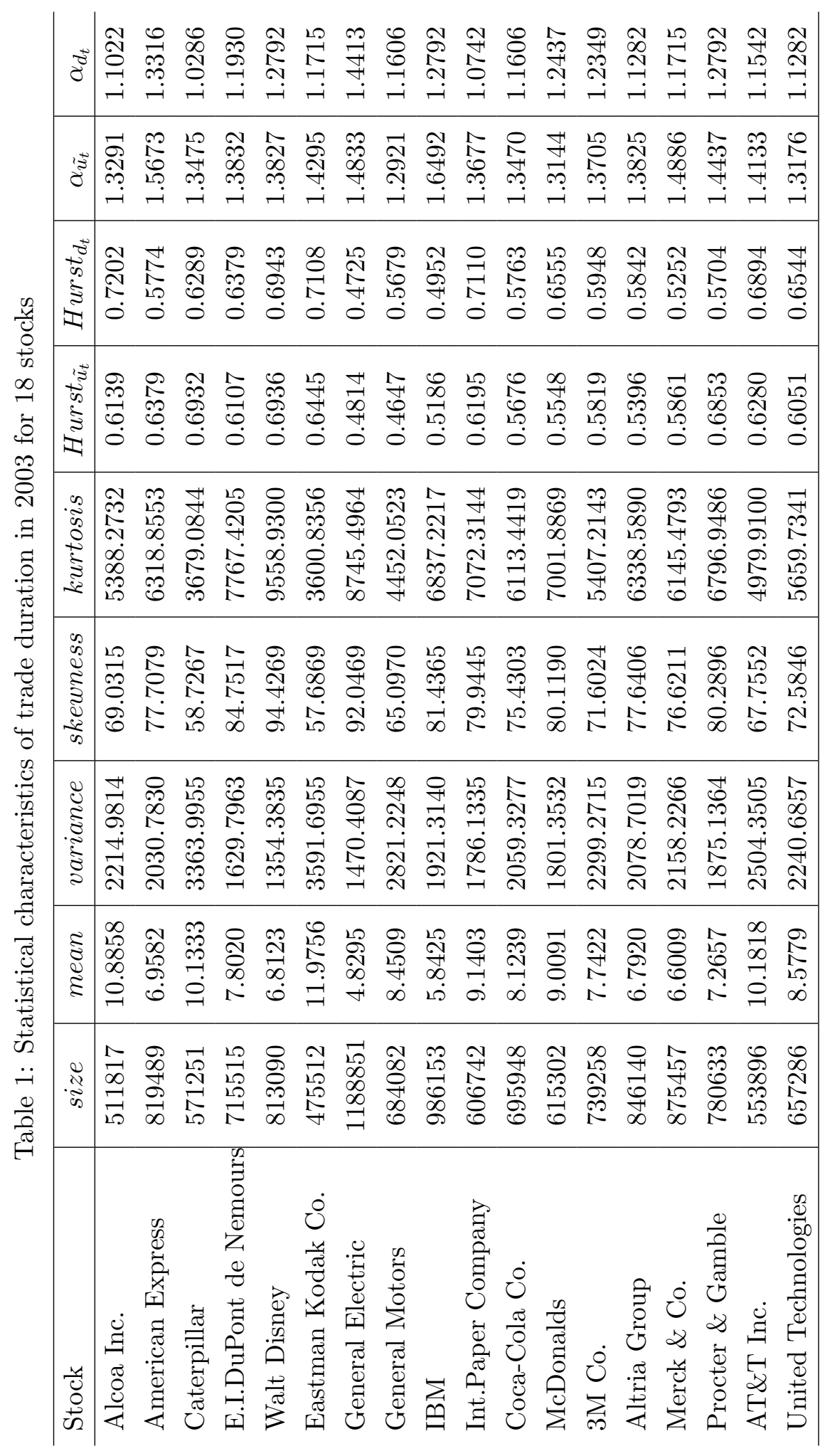




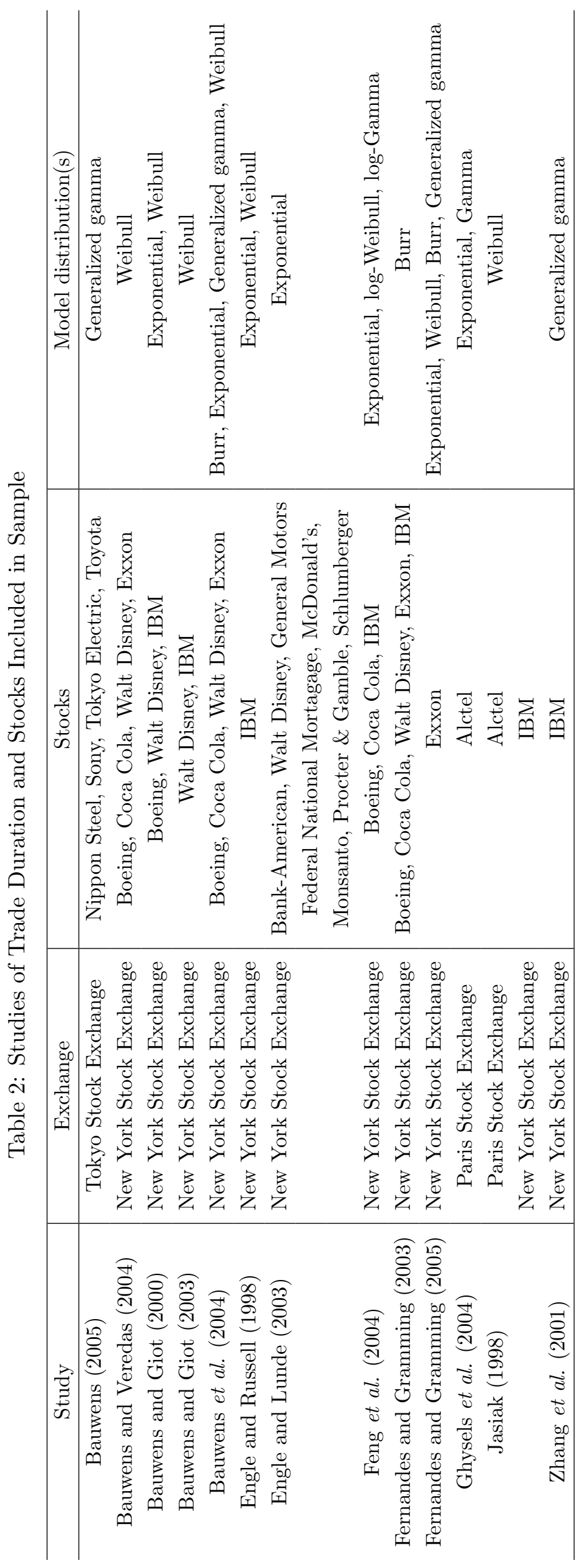


Table 3: ARCH-test for different lags of increments series generated from trade duration data. Test statistic and critical value are presented.

\begin{tabular}{c||c|c|c|c|c|c|c|c}
\hline Stock & lag1 & lag2 & lag3 & lag5 & lag10 & lag15 & lag25 & lag50 \\
\hline \hline Alcoa Inc. & 25020 & 33327 & 37483 & 41644 & 45423 & 46829 & 48008 & 48894 \\
American Express & 24976 & 33297 & 37441 & 41573 & 45281 & 46619 & 47668 & 48292 \\
Caterpillar & 24977 & 33322 & 37467 & 41629 & 45410 & 46814 & 47993 & 48880 \\
E.I.DuPont de Nemours & 24995 & 33329 & 37484 & 41647 & 45436 & 46851 & 48046 & 48970 \\
Walt Disney & 24999 & 33332 & 37490 & 41654 & 45439 & 46853 & 48045 & 48965 \\
Eastman Kodak Co. & 24967 & 33315 & 37466 & 41632 & 45417 & 46826 & 48012 & 48911 \\
General Electric & 24990 & 33328 & 37488 & 41653 & 45435 & 46848 & 48036 & 48945 \\
General Motors & 24987 & 33316 & 37463 & 41616 & 45377 & 46765 & 47909 & 48717 \\
IBM & 24967 & 33290 & 37430 & 41559 & 45256 & 46583 & 47614 & 48204 \\
Int. Paper Company & 24985 & 33325 & 37480 & 41645 & 45429 & 46841 & 48029 & 48936 \\
Coca-Cola Co. & 24981 & 33322 & 37480 & 41647 & 45430 & 46843 & 48036 & 48956 \\
McDonalds & 24978 & 33319 & 37479 & 41643 & 45426 & 46835 & 48019 & 48916 \\
3M Co. & 25138 & 33485 & 37667 & 41858 & 45667 & 47087 & 48284 & 49206 \\
Altria Group & 24990 & 33326 & 37484 & 41647 & 45429 & 46840 & 48026 & 48929 \\
Merck \& Co. & 24988 & 33325 & 37481 & 41648 & 45437 & 46852 & 48049 & 48978 \\
Procter \& Gamble & 25015 & 33332 & 37479 & 41639 & 45421 & 46833 & 48023 & 48937 \\
AT\&T Inc. & 24975 & 33320 & 37475 & 41642 & 45430 & 46843 & 48035 & 48953 \\
United Technologies & 24973 & 33318 & 37473 & 41639 & 45427 & 46839 & 48031 & 48950 \\
\hline \hline Critical Value & 3.8415 & 5.9915 & 7.8147 & 11.0700 & 18.3070 & 24.9960 & 37.6520 & 67.5050 \\
\hline
\end{tabular}


Table 4: Ljung-Box-Pierce Q-test statistic for different lags at $\alpha=0.05$. The italic numbers show the ratios of Q-test statistics compared with corresponding critical values.

\begin{tabular}{|c|c|c|c|c|c|c|c|c|}
\hline stock & $\operatorname{lag} 10$ & lag20 & lag50 & $\operatorname{lag} 100$ & $\operatorname{lag} 200$ & lag500 & $\operatorname{lag} 1000$ & $\operatorname{lag} 1500$ \\
\hline \multirow[t]{2}{*}{ Alcoa Inc. } & 760.3 & 931.7 & 1379.5 & 2041.0 & 3224.5 & 5912.9 & 9897.8 & $\overline{13327.0}$ \\
\hline & 41.5 & 29.7 & 20.4 & 16.4 & 13.8 & 10.7 & 9.2 & 8.4 \\
\hline \multirow[t]{2}{*}{ American Express } & 330.5 & 336.2 & 344.5 & 352.8 & 372.8 & 405.6 & 596.7 & 691.3 \\
\hline & 18.1 & 10.7 & 5.1 & 2.8 & 1.6 & 0.7 & 0.6 & 0.4 \\
\hline \multirow[t]{2}{*}{ Caterpillar } & 413.9 & 446.1 & 516.6 & 623.5 & 814.5 & 1329.9 & 2093.9 & 2899.9 \\
\hline & 22.6 & 14.2 & 7.6 & 5.0 & 3.4 & 2.4 & 1.9 & 1.8 \\
\hline \multirow[t]{2}{*}{ E.I.DuPont de Nemours } & 371.6 & 401.4 & 455.6 & 527.1 & 644.4 & 840.9 & 1184.5 & 1376.5 \\
\hline & 20.3 & 12.7 & 6.7 & 4.2 & 2.7 & 1.5 & 1.1 & 0.8 \\
\hline \multirow[t]{2}{*}{ Walt Disney } & 294.5 & 319.2 & 374.8 & 452.1 & 606.5 & 936.2 & 1475.7 & 1620.3 \\
\hline & 16.1 & 10.1 & 5.5 & 3.6 & 2.5 & 1.6 & 1.3 & 1.0 \\
\hline \multirow[t]{2}{*}{ Eastman Kodak Co. } & 157.9 & 177.6 & 204.4 & 257.0 & 351.2 & 654.6 & 1078.8 & 1530.8 \\
\hline & 8.6 & 5.6 & 3.0 & 2.0 & 1.5 & 1.1 & 1.1 & 0.9 \\
\hline \multirow[t]{2}{*}{ General Electric } & 386.8 & 391.1 & 393.1 & 394.8 & 397.4 & 406.6 & 601.1 & 627.5 \\
\hline & 21.1 & 12.4 & 5.8 & 3.1 & 1.6 & 0.7 & 0.5 & 0.3 \\
\hline \multirow[t]{2}{*}{ General Motors } & 350.8 & 356.2 & 365.7 & 373.3 & 396.8 & 513.5 & 584.7 & 832.1 \\
\hline & 19.1 & 11.3 & 5.4 & 3.0 & 1.6 & 0.9 & 0.5 & 0.5 \\
\hline \multirow[t]{2}{*}{ IBM } & 159.9 & 165.7 & 169.7 & 174.0 & 181.2 & 195.7 & 357.1 & 418.0 \\
\hline & 8.7 & 5.2 & 2.5 & 1.3 & 0.7 & 0.3 & 0.3 & 0.2 \\
\hline \multirow[t]{2}{*}{ Int. Paper Company } & 344.3 & 400.2 & 519.7 & 698.0 & 986.6 & 1424.1 & 1846.4 & 2299.0 \\
\hline & 18.8 & 12.7 & 7.6 & 5.6 & 4.2 & 2.5 & 1.7 & 1.4 \\
\hline \multirow[t]{2}{*}{ Coca-Cola Co. } & 244.2 & 253.4 & 266.0 & 293.4 & 318.8 & 366.1 & 620.3 & 758.9 \\
\hline & 13.3 & 8.0 & 3.9 & 2.3 & 1.3 & 0.6 & 0.5 & 0.4 \\
\hline \multirow[t]{2}{*}{ McDonalds } & 233.2 & 256.1 & 292.2 & 346.9 & 442.6 & 899.3 & 1016.4 & 1343.8 \\
\hline & 12.7 & 8.1 & 4.3 & 2.7 & 1.8 & 1.6 & 0.9 & 0.8 \\
\hline \multirow[t]{2}{*}{ 3M Co. } & 515.3 & 528.2 & 551.2 & 579.0 & 631.5 & 750.7 & 1044.4 & 1340.2 \\
\hline & 28.1 & 16.8 & 8.1 & 4.6 & 2.6 & 1.3 & 0.9 & 0.8 \\
\hline \multirow[t]{2}{*}{ Altria Group } & 279.1 & 284.2 & 292.6 & 299.0 & 319.1 & 357.7 & 556.0 & 617.1 \\
\hline & 15.2 & 9.0 & 4.3 & 2.4 & 1.3 & 0.6 & 0.5 & 0.3 \\
\hline \multirow[t]{2}{*}{ Merck \& Co. } & 262.9 & 268.0 & 276.1 & 280.0 & 285.9 & 303.0 & 461.6 & 535.6 \\
\hline & 14.3 & 8.5 & 4.0 & 2.2 & 1.2 & 0.5 & 0.4 & 0.3 \\
\hline \multirow[t]{2}{*}{ Procter \& Gamble } & 517.6 & 522.9 & 533.5 & 541.1 & 553.0 & 580.6 & 774.7 & 824.3 \\
\hline & 28.2 & 16.6 & 7.9 & 4.3 & 2.3 & 1.1 & 0.7 & 0.5 \\
\hline \multirow[t]{2}{*}{ AT\&T Inc. } & 280.2 & 319.1 & 398.1 & 517.9 & 691.1 & 990.2 & 1370.2 & 1709.4 \\
\hline & 15.3 & 10.1 & 5.8 & 4.1 & 2.9 & 1.7 & 1.2 & 1.1 \\
\hline \multirow[t]{2}{*}{ United Technologies } & 327.1 & 356.2 & 417.2 & 491.2 & 621.1 & 834.9 & 1130.4 & 1389.9 \\
\hline & 17.8 & 11.3 & 6.1 & 3.9 & 2.6 & 1.5 & 1.1 & 0.8 \\
\hline Critical Value & 18.3 & 31.4 & 67.5 & 124.3 & 233.9 & 553.1 & 1074.7 & 1591.2 \\
\hline
\end{tabular}




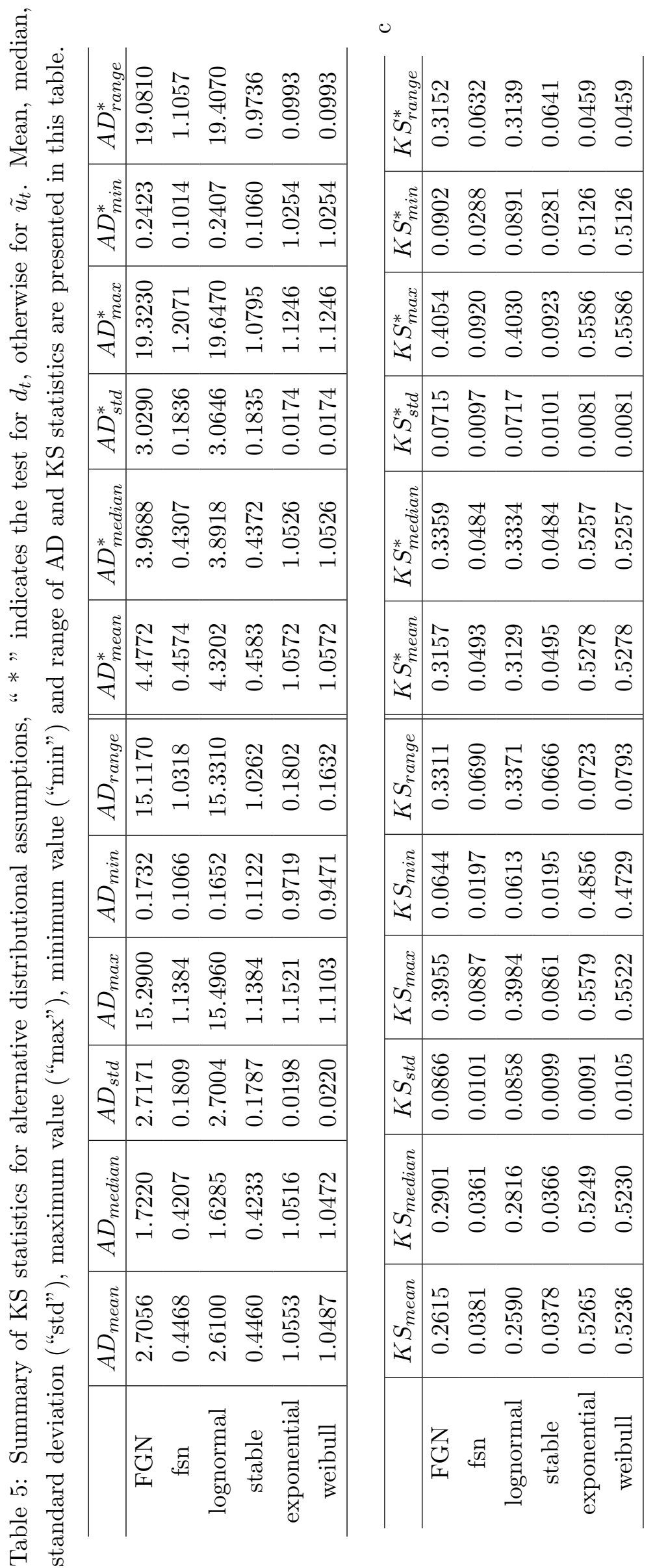


Table 6: Bootstrap 99\% confidence intervals for mean of differences in AD and KS statistics, "* * indicates statistics for $d_{t}$, otherwise for $\tilde{u}_{t}$. "FGN" stands for fractional Gaussian noise, "fsn" stands for fractional stable noise, "exp" stands for exponential distribution, "wbl" stands for Weibull distribution.

\begin{tabular}{c|c|c||c|c}
\hline$T:$ & $A D$ & $A D^{*}$ & $K S$ & $K S^{*}$ \\
\hline$E\left(T_{\text {stable }}-T_{F G N}\right)$ & $(-2.5223,-1.9851)$ & $(-4.3235,-3.7124)$ & $(-0.2318,-0.2157)$ & $(-0.2734,-0.2594)$ \\
$E\left(T_{\text {stable }}-T_{\text {lognormal }}\right)$ & $(-2.4281,-1.8893)$ & $(-4.1642,-3.5481)$ & $(-0.2292,-0.2132)$ & $(-0.2706,-0.2565)$ \\
$E\left(T_{\text {stable }}-T_{\text {exp }}\right)$ & $(-0.6278,-0.5913)$ & $(-0.6175,-0.5803)$ & $(-0.4895,-0.4878)$ & $(-0.4793,-0.4774)$ \\
$E\left(T_{\text {stable }}-T_{\text {wbl }}\right)$ & $(-0.6211,-0.5850)$ & $(-0.6175,-0.5804)$ & $(-0.4868,-0.4848)$ & $(-0.4793,-0.4773)$ \\
$E\left(T_{f s n}-T_{F G N}\right)$ & $(-2.5236,-1.9774)$ & $(-4.3204,-3.7077)$ & $(-0.2315,-0.2154)$ & $(-0.2736,-0.2597)$ \\
$E\left(T_{\text {fsn }}-T_{\text {lognormal }}\right)$ & $(-2.4265,-1.8888)$ & $(-4.1638,-3.5485)$ & $(-0.2290,-0.2130)$ & $(-0.2708,-0.2568)$ \\
$E\left(T_{f s n}-T_{\text {exp }}\right)$ & $(-0.6274,-0.5904)$ & $(-0.6182,-0.5815)$ & $(-0.4893,-0.4875)$ & $(-0.4795,-0.4776)$ \\
$E\left(T_{f s n}-T_{\text {wbl }}\right)$ & $(-0.6203,-0.5838)$ & $(-0.6185,-0.5815)$ & $(-0.4866,-0.4845)$ & $(-0.4795,-0.4776)$ \\
$E\left(T_{\text {fsn }}-T_{\text {stable }}\right)$ & $(-0.0057,0.0074)$ & $(-0.0077,0.0059)$ & $(-0.0002,0.0007)$ & $(-0.0007,0.0002)$ \\
\hline
\end{tabular}


Table 7: Supporting cases comparison of goodness of fit for fractional stable noise and stable distribution based on AD and KS statistics. Symbol "*" indicates the test for $d_{t}$, otherwise the test is for $\tilde{u_{t}}$. Symbol " $\succ$ " means being preferred and " " means indifference. Numbers shows the supporting cases to the statement in the first column and the number in parentheses give the proportion of supporting cases in the whole sample.

\begin{tabular}{c|c|c||c|c}
\hline & $A D$ & $A D^{*}$ & $K S$ & $K S^{*}$ \\
\hline fsn $\succ$ stable & 327 & 345 & 327 & 362 \\
& $(47.81 \%)$ & $(50.44 \%)$ & $(47.81 \%)$ & $(52.93 \%)$ \\
stable $\succ$ fsn & 344 & 328 & 351 & 318 \\
& $(50.29 \%)$ & $(47.95 \%)$ & $(51.32 \%)$ & $(46.49 \%)$ \\
fsn $\sim$ stable & 13 & 11 & 6 & 4 \\
& $(1.90 \%)$ & $(1.61 \%)$ & $(0.87 \%)$ & $(0.58 \%)$ \\
\hline
\end{tabular}
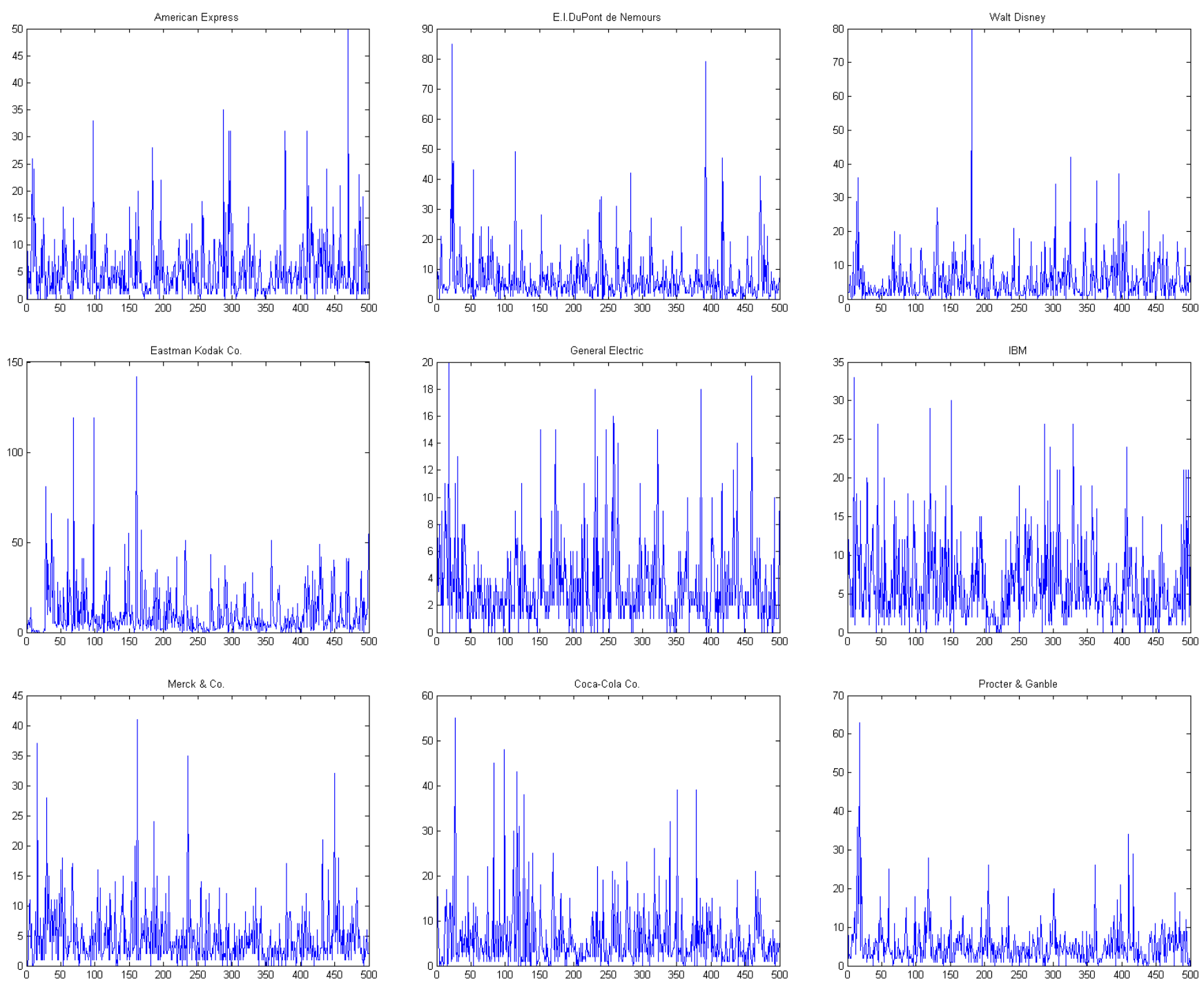

Figure 1: Plot of trade duration for several stocks. 


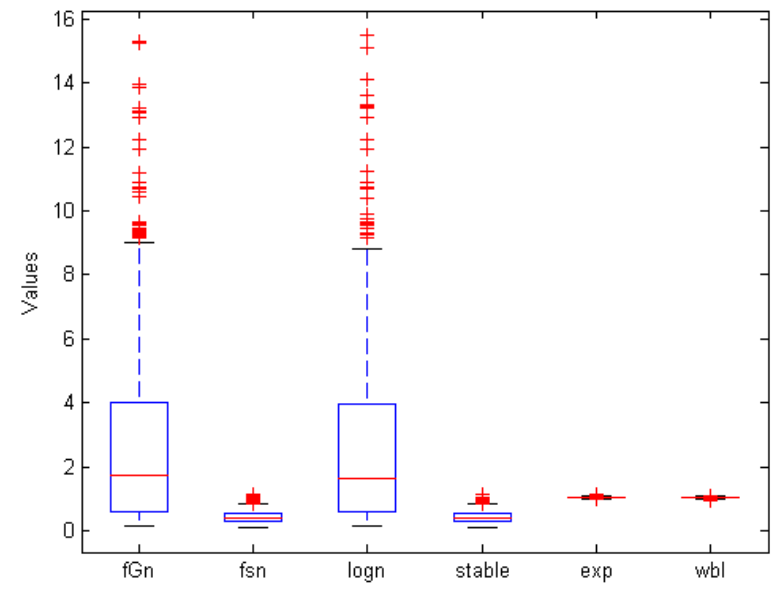

Figure 2: Boxplot of AD statistics for $\tilde{u}_{t}$ in alternative distributional assumptions.

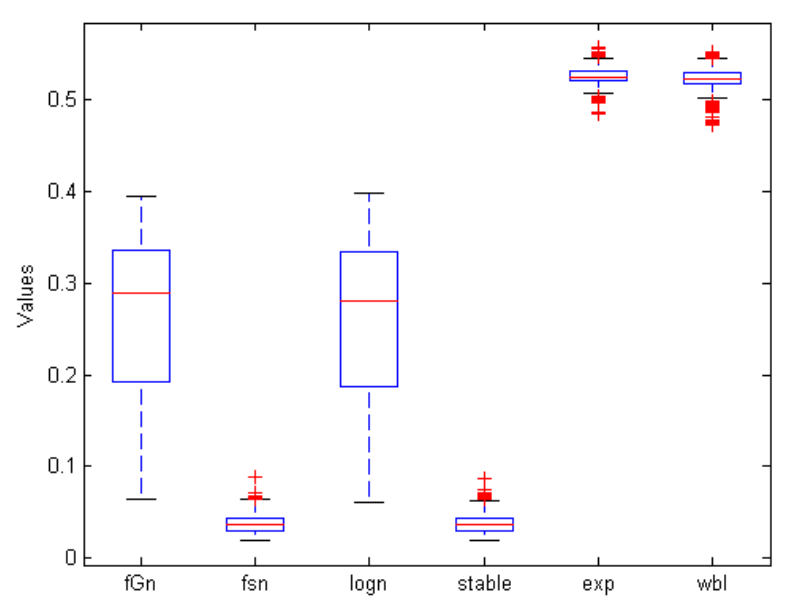

Figure 4: Boxplot of KS statistics for $\tilde{u}_{t}$ in alternative distributional assumptions.

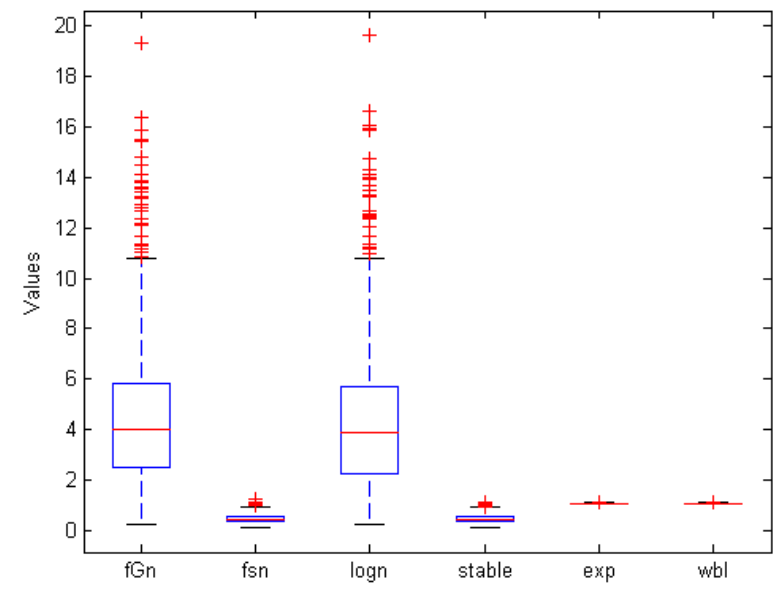

Figure 3: Boxplot of $\mathrm{AD}^{*}$ statistics for $d_{t}$ in alternative distributional assumptions.

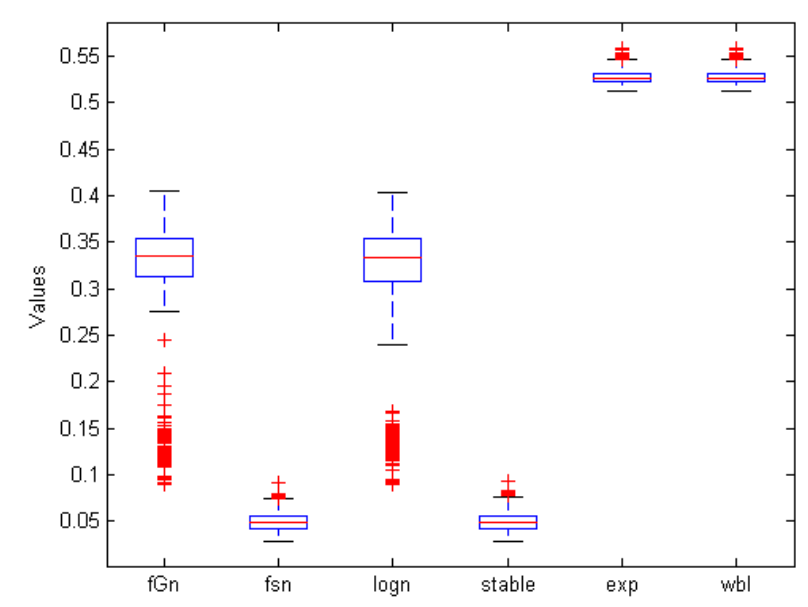

Figure 5: Boxplot of $\mathrm{KS}^{*}$ statistics for $d_{t}$ in alternative distributional assumptions. 